\title{
LA CARTA COMO ABSTRACCIÓN, CONOCIMIENTO Y DESENCUENTRO. EL CASO DE G.W. LEIBNIZ
}

\section{THE LETTER AS ABSTRACTION, KNOWLEDGE AND DISAGREEMENT THE CASE OF G.W. LEIBNIZ}

\author{
JUAN A. NiCOLÁs ${ }^{1}$ \\ Facultad de Filosofía y Letras. \\ Universidad de Granada
}

\begin{abstract}
Resumen: En la producción filosófica de los siglos XVI al XVIII ocupa un papel muy importante la correspondencia entre los intelectuales de diversas disciplinas. Dada la ausencia de revistas científicas, de editoriales y, por supuesto, de correo electrónico, la correspondencia postal (al ritmo posible en aquellos momentos) era un elemento clave de intercambio de conocimientos, de discusión y descubrimiento de nuevos hallazgos.

Se plantea aquí una reflexión sobre el sentido de la carta y su papel en la constitución del saber. Se destacan tres rasgos principales: la carta es una abstracción respecto a su contexto propio que es el conjunto de la correspondencia entre dos o más autores; la carta es un producto fundamental no sólo en la difusión del saber entre especialistas, sino también en su descubrimiento y creación; y por último, el intercambio epistolar no sólo constituye un modo esencial de comunicación entre científicos, políticos o intelectuales, sino que también a veces ha constituido la plataforma en la que han tenido lugar desencuentros de alcance histórico.
\end{abstract}

\footnotetext{
${ }^{1}$ Departamento de Filosofía II: Filosofía, Universidad de Granada, Campus La Cartuja, Edif. Psicología, 18071, Granada. E-mail: jnicolas@ugr.es
} 
Toda esta reflexión se ilumina al hilo de los textos y ejemplos de la inmensa correspondencia de Leibniz.

Palabras clave: Carta, correspondencia, Leibniz, Newton, conocimiento, desencuentro.

АвsтRACT: Correspondence between intellectuals of different disciplines occupied a very important place in philosophical production between the $16^{\text {th }}$ and $18^{\text {th }}$ centuries. The lack of scientific journals, publishers and email, made postal mail (at the speed possible at that time) one of the key factors for exchanging knowledge, discussion and discovering new findings. In this paper a reflection on the relevance of the letter in the construction of knowledge is presented. Three main features stand out. First, the isolated letter is an abstraction regarding its own context, the set of correspondence between two or more authors. Second, the letter is a fundamental product, not only for disseminating knowledge among specialists, but also for its discovery and creation. Finally, the exchange of letters is not only an essential means of communication between scientists, politicians and intellectuals, but has also sometimes been the platform where confrontations of historical significance have taken place. All these reflections are illustrated through texts and examples from the vast correspondence of Leibniz.

KeYwords: Letter, correspondence, Leibniz, Newton, knowledge, misunderstanding.

\section{Carácter poliédrico de "carta"}

"Carta" es una noción polisémica, plurifuncional y relacional. En primer lugar, el término significa objetos muy diversos. Puede referirse desde un objeto que permite un determinado juego (p.e., una carta de la baraja) hasta un argumento (por ejemplo, "Clinton jugó sus cartas en el debate con los demás líderes políticos"). Pasando por significados tales como objeto que informa a otro de algo (p.e., una carta de un banco a su cliente) o permiso para actuar con libertad (p.e, "La asamblea ha dado carta blanca al Presidente para decidir"). Incluso el término "carta" puede expresar ciertas habilidades (p.e, "España jugó sus cartas en el partido contra Holanda") o cualidades (p.e., "El director supo jugar sus cartas en la negociación”). 
Por otro lado, desde el punto de vista funcional, también la noción de "carta" admite una pluralidad prácticamente irreductible. Así por ejemplo, una carta se puede utilizar con el fin de adivinar el futuro de una persona (p.e., "le echaron las cartas y cambió su vida"). O bien se puede usar para orientarse en el mar (p.e., una carta de navegación); o para transmitir información (p.e., una carta de despido de un trabajador) o para difundir opiniones (p.e, una "carta al director" en un periódico), o para transmitir sentimientos (p.e, una carta entre amantes).

Toda esta diversidad de significados y funciones es de tal envergadura que resulta difícil encontrar rasgos comunes que permitan establecer una mínima univocidad. Uno de esos rasgos, aunque sea de tipo formal, podría ser el carácter relacional del término.

La carta, por último, en cuanto institución social que constituye un cierto "género literario" (tan diverso como se acaba de ver) contiene una lógica interna que implica una duplicidad de sujetos. Sea una carta entre amigos, o sea una carta profesional o una carta pastoral o una carta de citación judicial, hay alguien que expresa algo a alguien. Esto forma parte del sentido de la carta, por ello una carta que realmente se dirija a nadie se sitúa en el límite o bien del sinsentido o bien del campo semántico que la constituye.

Se va a analizar la estructura relacional de la carta y sus múltiples implicaciones de la mano de uno de los intelectuales que probablemente más cartas ha escrito a lo largo de la historia. Se trata del filósofo G.W. Leibniz (1646-1716). A lo largo de su obra puesta en forma de cartas se irán revisando tres aspectos: el aspecto lógico de la carta como abstracción, el aspecto epistemológico de la carta como medio de discusión y creación de conocimiento y el aspecto psico-político de la carta como desencuentro.

\section{La carta como abstracción}

La estructura interna relacional de la carta implica que una carta forma parte de un conjunto más amplio dentro del cual adquiere su sentido. Siendo así, una carta individual no es más que una parte de un todo, fuera del cual no cumple su función propia ni expresa aquello para lo que ha sido creada. Una carta es una pieza que encaja con una respuesta por parte del destinatario, incluso aunque éste ofrezca como reacción el no contestar. Este ir y venir de la carta y su 
respuesta constituye una unidad pragmática que no se puede destruir si se quiere mantener el sentido.

Esta idea ha sido expresada recientemente en la película "The Correspondence" de Giuseppe Tornatore (2016). Resumidamente el argumento consiste en una relación entre los dos co-protagonistas que en un momento determinado se separan y continúan su relación "por correspondencia", aunque el formato utilizado para ello es Internet. Se intercambian mensajes de audio y video hasta que uno de los corresponsales descubre que el otro ha muerto. Después de mucho dolor y sufrimiento, el corresponsal vivo sigue recibiendo mensajes del otro interlocutor. Esto le lleva a multitud de averiguaciones, viajes, entrevistas y sobre todo a un desconcierto absoluto. Tras muchas indagaciones va descubriendo que los mensajes que va recibiendo en su ordenador habían sido programados (con la colaboración de varias personas) antes de su muerte por el otro corresponsal. Cuando esto queda claro, el protagonista vivo decide en un momento determinado desconectar definitivamente el mecanismo del "intercambio epistolar". Ese modo de correspondencia la estaba llevando al borde de la locura y la muerte. Asumida de modo efectivo la muerte del otro corresponsal, carece de sentido intentar mantener una "correspondencia".

He aquí un caso, expresado artísticamente mediante una película, en el que expresa de modo dramático hasta qué punto "carta" es una noción bipolar, relacional. De ahí se deriva que la consideración de una carta aisladamente es un artificio al que le faltan piezas. El conjunto de esas piezas se llama correspondencia. Una carta forma parte de una correspondencia que puede consistir en muchas cartas o incluso en una sola. La correspondencia sería la unidad semántica y pragmática con sentido en sí misma. Por lo que se puede decir que una carta, considerada al margen de su pertenencia a una correspondencia completa no es más que una abstracción.

En el caso de G.W. Leibniz, escribió a lo largo de su vida unas 20.000 cartas pertenecientes a correspondencias con más de 1.300 corresponsales de 16 países diferentes. Hubo correspondencias que se mantuvieron a lo largo de casi 40 ańos (p.e., con la Princesa Carolina o con G. Molanus). Mantuvo intercambio epistolar con los más relevantes intelectuales y personajes de su época pertenecientes a los más diversos ámbitos del saber y de la vida social y política. Así, por ejemplo, mantuvo correspondencia con científicos (I. Newton, S. Clarke, N. Hartsoeker, L. Bourguet, N. Remond...), con matemáticos (J. Bernoulli, B. de Volder, Ch. Huygens, Mariotte, Oldenburg, Ch. Wolff...), con biólogos (Schwammerdam, 
Stahl, ...), con lógicos (A. Arnauld, ...), con juristas (S. Pufendorf, H. Conring, ....), con filósofos (Ch. Wolff, N. Malebranche, B. Spinoza, Th. Hobbes, B. Des Bosses, P. Bayle...), con políticos (J.F. von Hessen-Rheinfelds, Boyneburg, el Duque Ernst August, la reina de Prusia, Sofía Carlota, Princesa Carolina... ), con teólogos (J.B. Bossuet, P. Pellison, G. Molanus, D. Jablonski... ), etc.

Las correspondencias de Leibniz no siempre se realizan entre dos individuos, sino que hay frecuentemente triangulaciones. Por ejemplo, la correspondencia entre Leibniz y Newton a través de Oldenburg; o la correspondencia entre Leibniz y J. Bernoulli a través de De Volder; o la correspondencia entre Leibniz y A. Arnauld a través de Ernst von Hessen-Rheinfels. Este recurso era frecuente en la época. Del mismo modo era frecuente que las cartas fueran copiadas y reenviadas, con comentarios, a otros interlocutores; las cartas tenían un cierto carácter público, más que privado, pues su objetivo era la discusión y difusión del saber en sus más novedosos desarrollos. Por esto, las correspondencias forman parte de las "obras completas" de los filósofos de esta época.

La correspondencia expresa de un modo propio y explícito el papel del diálogo en la acción racional. En primer lugar, esto no ha de confundirse con el carácter dialógico de la razón ni con la razón dialógica. Ésta no consiste en el hecho de que la razón se desarrolle mediante diálogos individuales, ni siquiera en el formato de intercambio epistolar.

La dialogicidad de la razón se concibe como una alternativa al solipsismo metodológico, y su tesis fundamental consiste en que un individuo sistemáticamente solo no puede alcanzar conocimientos verdaderos. Esta dimensión de la racionalidad pone de manifiesto la prioridad del carácter originariamente público de la razón. Sólo una vez socializado en un marco de categorías interpretativas del mundo es posible entrar en la discusión racional, por ejemplo, en forma de correspondencia. Sólo así es posible, incluso, la innovación en lo relativo a interpretaciones o sentido del acontecer en el mundo. Esta infraestructura racional se adquiere dialógicamente, mediante los procesos socialmente institucionalizados al efecto. Este plano de constitución racional es originario respecto a todo diálogo efectivo entre individuos o grupos.

En segundo lugar, es frecuente el uso del "diálogo" en diversos ámbitos de creación literaria (novela, filosofía, teatro, poesía, canción...) Este recurso literario consiste básicamente en que el autor de la obra construye a lo largo de la misma dos o más personajes que representan y mantienen puntos de vista 
diferentes y correspondientes cada uno a los diferentes puntos de vista que se quieren confrontar.

Ejemplos de este modo de escribir hay muchos a lo largo de la historia y en la actualidad. En el caso de Leibniz, utilizó este recurso en una de sus obras más polémicas y desarrolladas, a saber, los Nuevos ensayos sobre el conocimiento humano. Lo utilizó también en multitud de "diálogos" breves, tales como "Conversación de Filareto y Aristo", "Diálogo entre un teólogo y un misósofo", "Diálogo entre Poliandro y Teófilo", "Diálogo entre Teófilo y Polidoro", "Diálogo entre Carino y Teófilo", etc.

En los Nuevos ensayos aparecen dos personajes, Teófilo y Filaletes, que representan respectivamente las opiniones de Leibniz y de J. Locke. La obra consiste en una detallada discusión del libro Ensayos sobre el conocimiento humano publicado por Locke. Son un ejemplo de análisis y crítica filosófica y de confrontación entre dos puntos de vista parcialmente divergentes como son la versión lockeana del empirismo y la tan particular versión leibniziana del racionalismo ${ }^{2}$.

Ahora bien, aunque externamente estas obras tienen formato de diálogo y de confrontación entre puntos de vista diferentes, es evidente que la obra tiene un único autor. Este autor único expone por un lado su propia opinión, y además reconstruye desde su propio punto de vista la opinión que quiere discutir. Esta reconstrucción pertenece siempre al ámbito creativo- del autor de la obra, y con ello encierra sistemáticamente una divergencia entre dicho autor y el verdadero autor de la opinión sometida a prueba. Incluso con la mejor de las voluntades, esta divergencia puede llegar a ser significativa. No faltan los ejemplos históricos de imprecisión (e incluso de manipulación) en la reproducción de la opinión del otro (cfr. Guillén Vera, T., 1990, 72-84).

Así pues, las obras con estructura de diálogo no son verdaderos ejemplos de tal modo de acción racional. A diferencia de la correspondencia. En ella sí que hay realmente una confrontación entre puntos de vista (que no tiene por qué ser conflictiva) en la que cada autor defiende su propia opinión en directo. Es un modo explícito de diálogo, no verbal sino gráfico. Evidentemente los contenidos pueden ser tan diversos como es la capacidad expresiva y experiencial humana. Se pueden transmitir desde sentimientos hasta tesis científicas, desde dudas hasta

${ }^{2}$ Un análisis de esta obra en cuanto "diálogo" puede verse en T. Guillén Vera, "Los Nuevos Ensayos. Ensayo de un diálogo pretendido” (1990). 
interrogantes, desde situaciones hasta interpretaciones. La correspondencia es un modo privilegiado de acción comunicativo-dialógica.

Este modo de interacción comunicativa con tan diversos contenidos contiene una dimensión epistemológica. Uno de los resultados de la dinámica dialógica de la correspondencia es la formulación o descubrimiento de nuevos conocimientos.

\section{La carta como medio de discusión y creación del conocimiento}

La carta en cuanto unidad de una correspondencia puede constituir, cuando el tema lo permite, una estrategia de indagación, más o menos planificada, que puede conducir a nuevos conocimientos.

La conexión entre las cartas y el conocimiento no ha desaparecido completamente de nuestra cultura. "Echar las cartas a alguien" es un modo de acceder al conocimiento de lo que será su futuro en algún aspecto concreto.

Pero en el sentido de "carta" que estamos manejando aquí hubo momentos en la historia en que se constituyó en uno de los modos fundamentales de discusión entre científicos, filósofos, teólogos o juristas. Era la época anterior a Internet y a las revistas y congresos científicos. Hay correspondencias que contienen el proceso de argumentación que condujo a determinados descubrimientos.

Retomando el caso de Leibniz, en su obra se pueden encontrar multitud de correspondencias de contenido científico. En el siglo XVII comienzan a fundarse revistas y sociedades científicas con el fin comunicar los resultados de las investigaciones a la comunidad, poner en relación a los científicos y estimular la discusión y desarrollo de los experimentos e indagaciones. Leibniz fue pionero en el impulso de la idea de "Europa" como unidad cultural; y para ello fundó Academias (Academia de Ciencias de Berlín), fundó revistas, luchó por la unidad de las confesiones cristianas, etc.

Un caso paradigmático de la capacidad de la correspondencia como medio de discusión científica y de alcanzar nuevos conocimientos fue la correspondencia con Johan Bernoulli. Esta correspondencia se mantuvo durante 23 años, hasta 
la muerte de Leibniz y consta de 275 cartas $^{3}$. A lo largo de la correspondencia Leibniz discute con Bernoulli multitud de problemas matemáticos y entre ellos el correspondiente al modo de calcular la razón de procesos naturales de sucesión infinita. En el intercambio se van forjando conceptos como vórtices, continuidad, vacío, logaritmo, series infinitas, tangentes inversas, cuadraturas, etc. Hasta que van apareciendo con un significado nuevo y específico las nociones de infinitésimo, sumatorio, integral y diferencial. Había nacido en este contexto el cálculo diferencial. Consiste fundamentalmente en un supremo esfuerzo por reducir la serie infinita de la sucesión de fenómenos naturales a una razón matemática manejable. La aproximación entre ambas variables es una tendencia a cero, que nunca se alcanza pero que permite a la razón humana atisbar la inteligibilidad de lo infinito.

\section{La carta como desencuentro}

La correspondencia tiene un componente de encuentro con el destinatario que puede afectar a muy diversas esferas de la personalidad de los corresponsales, según la índole del contenido de las cartas. Pueden llegar a expresar y transmitir el más profundo enamoramiento, o incluso ser el escenario en que se produce (por ejemplo, vía correo electrónico).

Pero en el intercambio epistolar no siempre tiene lugar una feliz y fructífera interacción personal o temática. A veces el cruce de mensajes conduce al más profundo de los distanciamientos, malentendidos o enfrentamientos. Todos tenemos experiencia o conocemos casos de cartas que parten el corazón, o que significan una despedida definitiva, o que producen una incontenible tristeza y llanto, o que ponen de manifiesto una incompatibilidad irreductible.

Esta posibilidad (tantas veces real) no es algo tangencial o externo a la dinámica del intercambio postal, sino que forma parte de su más profunda lógica interna. He aquí la dimensión que la correspondencia encierra de desencuentro con el otro.

\footnotetext{
${ }^{3}$ La correspondencia entre Leibniz y Johan Bernoulli ha sido traducida completa por primera vez al castellano por B. Orio de Miguel en G.W. Leibniz, Obras filosóficas y cientificas, vols. 17A y 17B: Correspondencia III.
} 
Siguiendo con el caso de Leibniz, también hubo correspondencias de este tipo, no todas fueron apasionadas discusiones matemáticas o políticas con final feliz. Quizás el caso con más repercusiones históricas, científicas, filosóficas y políticas fue su intercambio con I. Newton. Esta correspondencia estuvo entretejida con la correspondencia con S. Clarke, defensor de la posición de Newton y otros que intervinieron en la discusión desde diversas posiciones. Así, tomaron parte en este intercambio, además de Newton, Leibniz y Clarke, la Princesa Carolina de Gales, Conti, Chamberlayne, Remond de Montmort, Keil, J. Bernoulli, etc.

La discusión entre Leibniz y Newton no sólo fue en torno a una cuestión matemática. Tenía un trasfondo metafísico (concepción del papel de Dios, noción de fuerza, de absoluto), científico (concepción del espacio y del tiempo), por supuesto matemático (cálculo diferencial e integral desde perspectiva matemática o físico-geométrica), y también político. Estaban en juego las relaciones entre la casa de Hannover (aún inexistente Alemania como tal) y la corona de Inglaterra. Leibniz estaba directamente relacionado con la casa de Hannover, de donde acababa de salir el rey de Inglaterra (Jorge I) y su esposa, la Princesa Carolina de Gales, posteriormente reina de Inglaterra. Por otro lado, Newton era presidente de la Royal Society, institución de alto prestigio científico. Este trasfondo es el que determina una polémica que se centró en la prioridad en la invención del cálculo infinitesimal y se desarrolló en medio de lo que hoy se consideran manipulaciones y falsedades desde el entorno de Newton, acusando a Leibniz de haber copiado a Newton. A estas alturas parece claro que ambos descubrieron el cálculo por vías diferentes (algo que Leibniz reconoció), y con signaturas y notaciones distintas. Pero no era principalmente una cuestión matemática lo que estaba en juego, ni tampoco es éste el lugar de discutir este aspecto de la polémica.

Lo que interesa aquí es que la carta como género literario y como medio de comunicación personal y profesional estuvo en medio de toda esta discusión. Y las acusaciones de ocultamiento de cartas, manipulaciones mediante ańadidos y recortes fueron el formato que adquirió la polémica.

Sin entrar en más detalles, resumidamente lo que ocurrió fue lo siguiente. En 1673-4 estando Leibniz en París se dedicó al estudio de series numéricas. En 1674 recibe una carta de M. Oldenburg diciéndole que el Sr. Newton ya había conseguido cosas semejantes, aunque el método de Leibniz "fue muy aplaudido 
por Newton" ${ }^{4}$. En 1676 llega Leibniz a la formulación del cálculo diferencial, año en que pasa por Londres a su vuelta de Francia a Hannover. Al llegar a Hannover, Leibniz recibe una carta de Newton (a través de Oldenburg) donde decía que disponía de dos métodos para hallar la figura de las tangentes; pero estos métodos fueron "encriptados" por Newton, de modo que no había una exposición de los mismos. En respuesta, Leibniz envía a Newton en carta de junio de 1677 su método, que según Leibniz podía conseguir las mismas cosas que Newton decía, aunque éste no lo había mostrado claramente.

Posteriormente, Leibniz publicó en el Journal des Savans de Leipzig en 1682 y 1684 sus avances sobre series para el círculo y sobre el cálculo de diferencias, "que había inventado y guardado casi nueve años sin preocuparme por publicarlo".

En 1687 I. Newton publicó sus Principia Mathematica en la que dice:

En el intercambio de cartas que mantuve hace ya diez años con el Sr. Leibniz, muy hábil geómetra, donde le hacía saber que tenía un método para determinar las cantidades más grandes o las más pequeñas, de hallar las tangentes... que yo escondí bajo letras transpuestas... este célebre personaje me respondió que él había dado con un método que también hacía esto y lo comunicó; el cual no difería apenas del mío más que en los términos y en los signos.

Este texto lo comenta Leibniz diciendo que Newton no protestó en absoluto cuando le comunicó que había alcanzado el método por sí mismo. Y por otro lado, en la carta a Conti de abril de 1716 dice que "no hay el menor rastro ni sombra del cálculo de diferencias o fluxiones en todas las antiguas cartas del Sr. Newton que yo haya visto, excepto en la del 24 de octubre de 1676, en donde él sólo habla en enigma, y la solución de este enigma que no ha dado hasta diez años más tarde”.

Pero a partir de ahí, en 1711, según Leibniz "hubo gentes en Inglaterra que azuzados, a lo que parece, por sentimientos de envidia" se lanzaron a disputar la prioridad de la invención del cálculo diferencial. Se publicó por parte de la Royal Society (de la que I. Newton era presidente), sin conocimiento de Leibniz, un

\footnotetext{
${ }^{4}$ Carta de Leibniz a la Baronesa de Kilmansegge de 7 de abril de 1716. Las cartas mencionadas a continuación están recogidas en Obras filosóficas y científicas de G.W. Leibniz, vol. 18: Correspondencia $V$. La traducción es de Eloy Rada.
} 
libro titulado Commertium Epistolicum en el que se recogían las cartas intercambiadas por ambos autores y otros documentos y comentarios de los editores. Ahí se acusó a Leibniz de querer asumir la prioridad en el descubrimiento del cálculo diferencial. Todo dependía de cómo traducir e interpretar una frase de un trabajo publicado por Leibniz en las Acta Eruditorum de enero de 1705 que decía: "Pro differentiis leibnitianis D. Newtonus adhibet, semperque adhibuit fluxiones". Esta es la frase de la discordia. En el libro Commercium Epistolicum los editores, del círculo de Newton, interpretaron que Leibniz reclamaba la prioridad sobre Newton en el descubrimiento del cálculo diferencial (cálculo de fluxiones en la terminología de Newton): "el sentido de las palabras es que Newton sustituyó las fluxiones por las diferencias leibnizianas" Leibniz. Pero Leibniz rechaza esta interpretación en carta a Conti de 9 abril de 1716 diciendo que". "esta es una interpretación maliciosa de una persona que busca pendencia... no es en modo alguno que después de haber visto mis diferencias, sino que desde antes él se ha servido de las fluxiones. Y desafío a quien quiera que sea a dar otro sentido razonable a estas palabras, semperque adhibuit

Pero el mal ya estaba hecho, porque "el Sr. Newton lo ha hecho publicar en el mundo mediante un libro impreso para desacreditarme y lo ha enviado a Alemania, a Francia y a Italia, como en nombre de la Royal Society".${ }^{6}$ Este libro se imprimió y se difundió sin que Leibniz tuviera noticia de ello.

Por su parte, Leibniz defendió que nunca había dicho que Newton elaborara el cálculo de fluxiones a partir de su cálculo diferencial, pero que era igualmente falso que él hubiera tomado las ideas de Newton para llegar al cálculo diferencial. Su postura fue pensar que ambos descubrieron el cálculo diferencial por distintas vías. En la carta a Conti de abril de 1716 dice:

No es por las fluxiones de líneas sino por las diferencias de números como yo he llegado finalmente a considerar que estas diferencias aplicadas a las magnitudes que crecen continuamente se desvanecen en comparación con las magnitudes diferentes, en lugar de permanecer como hacen en las sucesiones de los números. Y creo que esta vía es la más analítica, el cálculo geométrico de las diferencias que es el mismo que el de las fluxiones".

\footnotetext{
${ }^{5}$ Carta de Leibniz a Conti de 9 de abril de 1716.

${ }^{6}$ Carta de Leibniz a Chamberlayne de 17 de abril de 1714.
} 
En la carta a Chamberlayne de 1714 escribe: "Yo siempre he hablado como si de su cosecha él [Newton] hubiera sabido cierta cosa semejante a mi método". Y en carta a la Baronesa de Kilmansegge de abril de 1716 dice: "Yo he tenido la honestidad de decir públicamente, y hacer decir a mis amigos, que yo creía que el Sr. Newton había tenido de su propia cosecha cierta cosa similar a mi invención”.

En la disputa se le pidió su opinión a J. Bernoulli, prestigioso matemático del momento, y dijo en carta que después recogió Leibniz en su carta a la Baronesa de Kilmansege de 7 de abril de 1716:

Mi conjetura se apoya en un indicio muy fuerte. Es que en todas las cartas del Commertium Epistolicum no se encuentra la menor traza ni sombra de letras como $x$ o $y$, puntuadas por uno, dos, tres o más puntos sobrepuestos, que ahora emplea en lugar de $d x, d d x, d d d x, d y, d y, d d y$, etc. E incluso en la obra de los Principia Mathematica, donde tenía tan frecuentemente ocasión de emplear su cálculo de fluxiones, no dice una palabra, y no se ve ninguna de estas señales... Estas letras puntuadas no han aparecido hasta el tercer volumen de las Obras del Sr. Wallis [en 1693], muchos años después de que el cálculo de diferencias hubiese sido recibido por todos.

Leibniz comparte esta misma opinión, que expresa en la Charta Volans, considerada de su autoría:

El Sr. Newton no había llegado aún a conocer el verdadero cálculo de diferencias en 1687, cuando publicó su libro Principia Mathematica, pues además de que no lo hizo aparecer, aunque tuvo muy buenas ocasiones para ponerlo en práctica, cometió errores que no son compatibles con el conocimiento de este cálculo.

En la carta a Conti de abril de 1716, Leibniz llega a decir sobre la prioridad del descubrimiento: "Si la cuestión consistiera únicamente en quién de los dos, el Sr. Newton o yo, halló primero el cálculo en cuestión, no me hubiera preocupado 
en absoluto. Pues es difícil decidir lo que uno y otro puede haber guardado in petto y durante cuánto tiempo. Pero un seguidor del Sr. Newton ha pretendido que yo lo había tomado de él”. Esa fue la idea difundida en el Commertium Epistolicum, y por eso Leibniz se ve obligado a cambiar de opinión respecto a Newton: "El Sr. Newton dice que yo lo he acusado de ser un plagiario, pero ¿dónde está el lugar en que lo he hecho? Son sus incondicionales quienes han parecido intentar esta acusación contra mí, y él ha entrado en connivencia con ellos". Y en la carta Chamberlayne dice: "Pero engañado [Newton] por algunos de sus aduladores malinformados se ha dejado llevar a atacarme de manera muy sensible".

Así, Leibniz se siente injustamente tratado ("mi honestidad ha sido mal reconocida", "parece que se buscaba un pretexto de ruptura") y pasa de "estar muy inclinado a creer al Sr. Newton por su palabra" a una opinión mucho más precavida y desconfiada: dado "su último proceder, me ha obligado a ser mucho más cauto a este respecto". Y en la carta a Conti mencionada: "Tengo una tan gran opinión sobre el candor del Sr. Newton que le he creído bajo su palabra, pero al verle en connivencia con las acusaciones cuya falsedad le es conocida, era natural que empezase a dudar".

La ruptura estaba consumada y además de forma definitiva. En carta de 29 de noviembre de 1716, Conti comunicaba a Newton: "El Sr. Leibniz ha muerto y la disputa ha terminado".

Independientemente de la aclaración del fondo de la disputa, si es que fuera posible para la ciencia histórico-filosófica, en este contexto lo relevante es que se trata de toda una polémica científica, filosófica, matemática mantenida fundamentalmente en el formato de correspondencia en la que se cruzaron cartas de diversos corresponsales como Oldenburg, Conti, Bernoulli, Clarke, Princesa Carolina, Baronesa de Kilmansegge, etc., además de Leibniz y Newton. Todo este trasiego de cartas en diversos idiomas, países y ámbitos del saber acabaron en una desavenencia de alcance histórico.

He aquí un caso en el que el intercambio epistolar acabó en la incomprensión mutua y en la imposibilidad de llegar a un acuerdo. Es la posibilidad que la correspondencia contiene de desencuentro con el otro. 


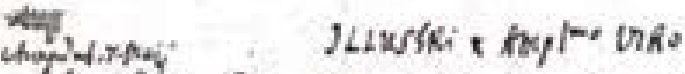

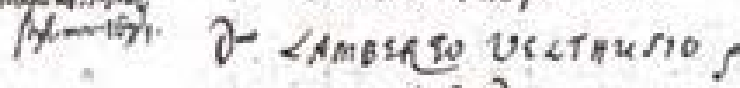

$$
\begin{aligned}
& \text { J.P. } \partial \text {. }
\end{aligned}
$$

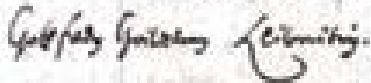

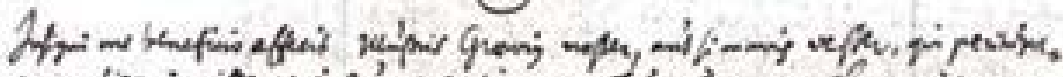

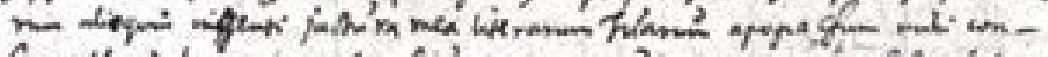

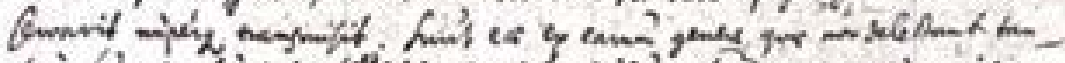

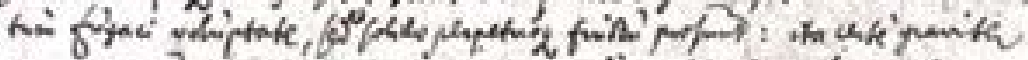

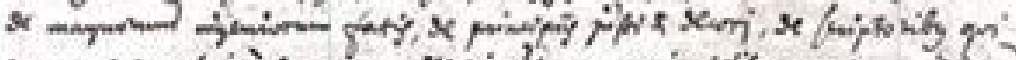

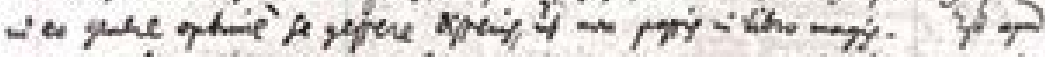

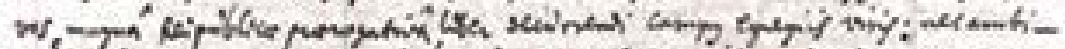

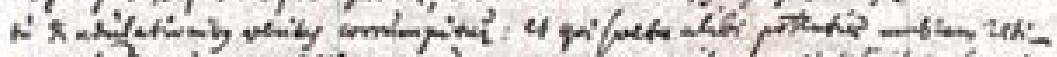

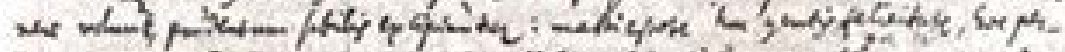

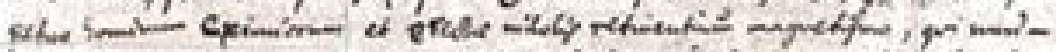

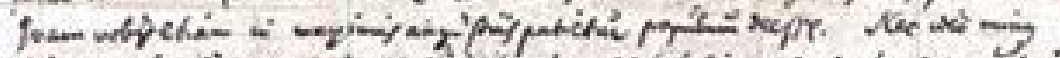

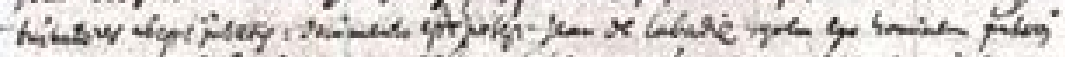

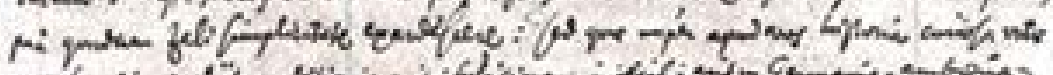

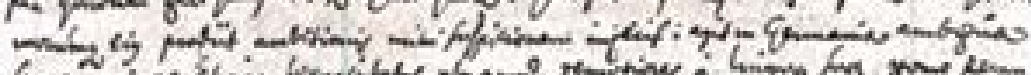

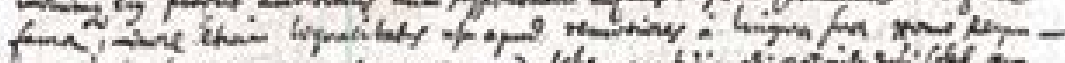

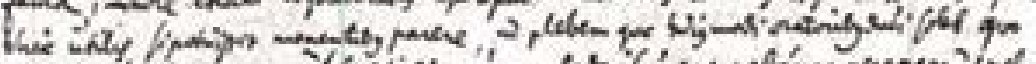

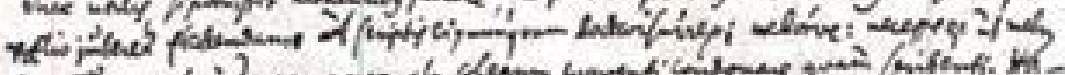

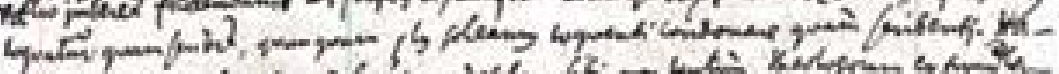

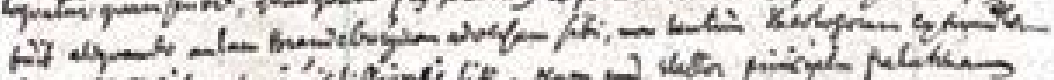

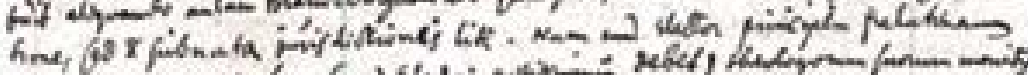

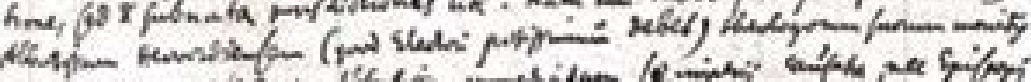

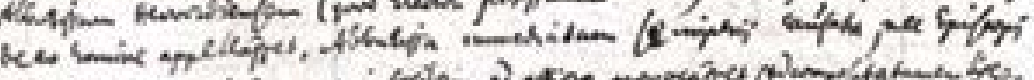

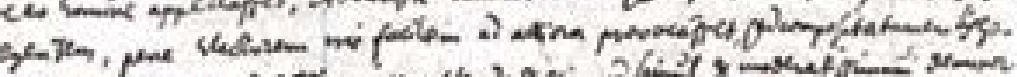

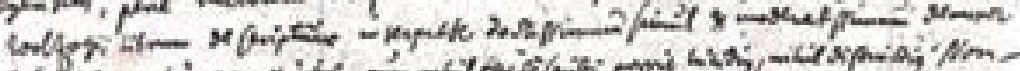

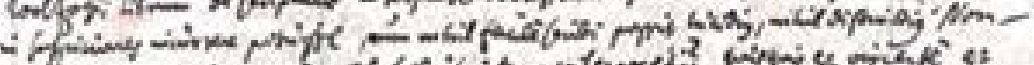

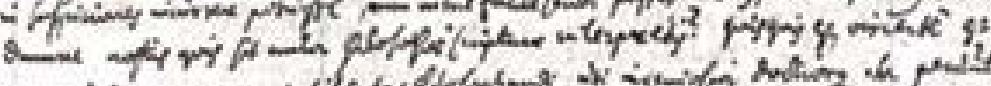

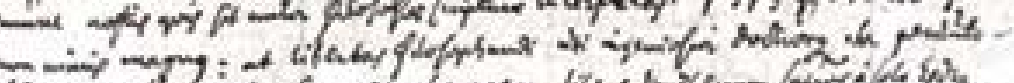

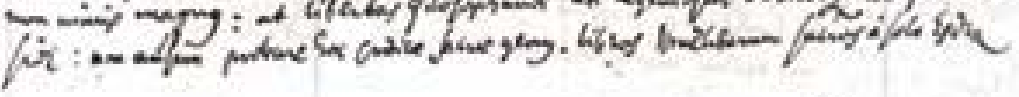

Carta de G.W. Leibniz a L. Velthius 


\section{Bibliografía}

- Leibniz, G.W. (2011), Obras filosóficas y científicas, trad. B. Orio de Miguel, vols. 17A y 17B: Correspondencia III, ed. Comares, Granada.

- Leibniz, G.W. (2016), Obras filosóficas y cientificas, trad. E. Rada, vol. 18: Correspondencia $V$, ed. Comares, Granada.

- Cubells, M.R. (en prensa), "La correspondencia de Leibniz en el conjunto de la obra leibniziana" en J.A. Nicolás, M. Mendonça (eds.), Guia Comares de Leibniz (Monadologia), ed. Comares, Granada.

- GäDEKE, N. (2009), "Leibniz lässt sich informieren. Asymmetrien in seinen Korrespondenzbeziehungen", en K.D. Herbst, S. Kratochwil (Hrsg.), Kommunikation in der frühen Neuzeit, P. Lang Verlag, Frankfurt a.M., 25-46.

- Guillén Vera, T. (1990), "Los Nuevos Ensayos. Ensayo de un diálogo pretendido", Azafea, 3, 63-86.

- Taton, R. (1976), "Le rôle et l'importance des correspondences scientifiques aux XVIIe et XVIII siècles", Revue de Synthèse, 81-82, 7-22.

- Ütermöenlen, G. (1976) "La correspondence de Leibniz et son édition dans les 'Sämtliche Schriften und Briefe", Revue de Synthèse, 81-82, 95-106.

Recibido: 1/09/2016

Aceptado: 20/10/2016

Este trabajo se encuentra bajo una licencia de Creative Commons Reconocimiento-

NoComercial-SinObraDerivada 4.0 Internacional 
